\title{
Un cas de stomatite nécrosante : noma des pays riches?
}

\author{
Sylvain Catros, Sébastien Lerici, Jean-Christophe Fricain, \\ Jean-Marie D'Elbée, Rémy Gaston, Nicolas Glock,
}

\author{
UFR d'Odontologie, 16-20 cours de la Marne, 33000 Bordeaux, France
}

seblerici@gmail.com

\begin{abstract}
Dans les pays développés, les cas d'ostéonécrose maxillaire spontanée sont essentiellement liés à l'utilisation de bisphosphonates administrés par voie intra-veineuse (Ruggiero 2011). Quelques cas de nécroses osseuses maxillaires avec expulsion dentaire spontanée ont été décrits au cours d'infections virales à herpes zoster (Mintz et Anavi 1992). Le noma (cancrum oris) touche préférentiellement les tissus mous (Enwonwu et al. 2006). Il est endémique dans les pays pauvres, où le défaut d'hygiène et la malnutrition font le lit de cette gangrène (Baratti-Mayer et al. 2003).
\end{abstract}

Le cas présenté concernait une femme de $45 \mathrm{ans}$, initialement suivie pour une connectivite mixte non étiquetée. Les traitements successifs ont comporté de l'Endoxan ${ }^{\circledR}$, du Cellcept ${ }^{\circledR}$, puis du Rèrituximab, systématiquement associés à une corticothérapie au long cours. Face à l'inefficacité de ces traitements, un traitement associant méthotrexate et du Remicade ${ }^{\circledR}$ a été prescrit à la patiente. L'apparition d'ulcérations buccales secondaires à la prise de méthotrexate a fait suspecter ce médicament, initialement prescrit à la posologie de $20 \mathrm{mg}$ par semaine. Malgré un arrêt du méthotrexate, les ulcérations se sont étendues et ont évolué vers une nécrose osseuse avec exfoliation spontanée des incisives maxillaires et de la première molaire maxillaire gauche. Une antibiothérapie probabiliste $\left(\right.$ Fortum $^{\mathbb{R}}-$-Vancomycine-Flagyl ${ }^{\mathbb{B}}-$ Amiklin $^{\circledR}$ ) a été instaurée. Devant la dénutrition objectivée par une hypoalbuminémie et un indice de masse corporelle de 15,3, une nutrition parentérale a aussi été mise en place. Une amélioration de l'état général a rapidement été observée. Dans la cavité buccale, l'extension du processus a abouti en trois semaines à l'élimination de la muqueuse palatine et à l'exposition osseuse du 1/3 antérieur du palais et de la mandibule au niveau de 46-48. Les canines maxillaires mobiles ont été avulsées. La patiente ayant été récusée pour une ablation de l'os nécrosé et reconstruction par lambeau, une résection osseuse modelante et une prothèse obturatrice ont été réalisées.

On ne retrouve pas dans la littérature de cas de nécrose osseuse spontanée associée à un traitement immuno-suppresseur. Les rares cas de nécroses maxillaires avec exfoliation dentaire ont été décrits dans l'infection avec le virus de la varicella et du zona (Lambade, 2011). Chez cette patiente, dans les prélèvements bactériens, fongiques et viraux, on n'a retrouvé que Pseudomonas aeruginosa comme agent infectieux potentiel à l'origine des manifestations cliniques chez cette patiente. Plusieurs cas d'ostéites secondaires à la surinfection de lésions cutanées par Pseudomonas aeruginosa ont été rapportés (Elouennass 2007) mais jamais au maxillaire.

Chez cette patiente, la nécrose osseuse spontanée est apparue dans un contexte d'immunodépression liée à sa pathologie, à ses traitements et à sa dénutrition. Malgré une prise en charge précoce, la nécrose s'est étendue à la muqueuse et à l'os en préservant les tissus mous de recouvrement. Face à ce tableau clinique, se pose la question de l'étiologie de la nécrose, et de la mise en cause possible du méthotrexate ou du Remicade ${ }^{\circledR}$. Le contexte de dénutrition et l'évolution favorable après mise en place de la nutrition parentérale font évoquer une forme de noma, mais l'absence d'évolutivité vers les tissus mous musculo-cutané ne concorde pas avec la description classique. 